\title{
The Red Dwarf Stars of the UV Ceti-Type in the Neighbourhood of the Sun
}

\author{
N. I. SHAKHOVSKAYA (Crimea)
}

(Read by E. SCHÖFFEL)

The intensive patrol observations of red dwarf stars during last years, carried out in the Crimea, Chile, Catania, South Africa, Armenia, Japan and other observatories led to appreciable increase of number of known UV Ceti-type objects. It permits us to complete the known lists of the variables $(1-4)$. At the same time it is worth-while to define more precisely the criteria to consider a star as the UV Ceti-type variable, and to carry out strong selection of the certain UV Cet-type variables from the objects suspected to be this type variables.

The following definition of the UV Cet-type variables was given in (5): „dMe stars, sometimes subjected to flares with the amplitude from $1^{\mathrm{m}}$ to $6^{\mathrm{m}}$. Maximum brightness is attained in seconds or dozens of seconds after the commencement of the flare, the star returns to the its normal brightness after several minutes, or dozens of minutes. A typical representative is UV Ceti." Now it is impossible to consider this definition as a quite right because: 1) There are a number of $M$-dwarf stars affected by the flares similar to the UV Ceti flares, but in their quiet state spectra no emission lines are observed. For example, BD $+43^{\circ} 44 \mathrm{~A}, \mathrm{BD}+43^{\circ} 44 \mathrm{~B}$ and SZ UMa, the flare activity of these stars were detected in Crimea; and probably,

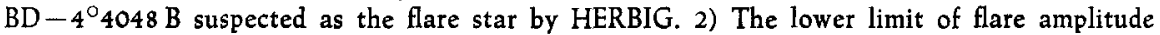
cited in (5) corresponds to visual observations, but the modern photoelectric observations register flares with amplitudes to omo2-0mo5. Therefore we suppose, that UV Cet-type variables are K-M dwarfs, which show quick flares with amplitudes exceeding the observational errors, and duration of the flares are from a few seconds up to a few hundred minutes.

Table 1 includes only such variable K-M dwarf stars, for which existing observations allow to construct flare light curves. All stars in Table 1, except V 371 Ori, have photoelectric flare light curves. The strong flare of V 371 Ori was observed in radio region, but simultaneous optical observations were carried out photographically and visually only.

Table 1 contains following data: 1) the number from the catalogue (6); 2) names from $(5)$; 3) the designations from the other catalogues; 4-5) Right ascention and declination for 1950.0 ; 6) spectral type; 7-8) V, B-V - the apparent magnitude and the colour; 9) the absolute magnitude $M_{v}$. All data in the columns $1-9$ were taken from (5-7). In two last columns bibliography of original investigations or surveys is given: in the column 10 for the flares photometric observations and in the column 11 for the flares spectral observations. For close double stars a symbol $\mathrm{J}$ in columns $7-10$ denotes that the published photometry and the flare observations were carried out for the combined light. An asterisk in the column 1 means that there is a note after the Table 1. 


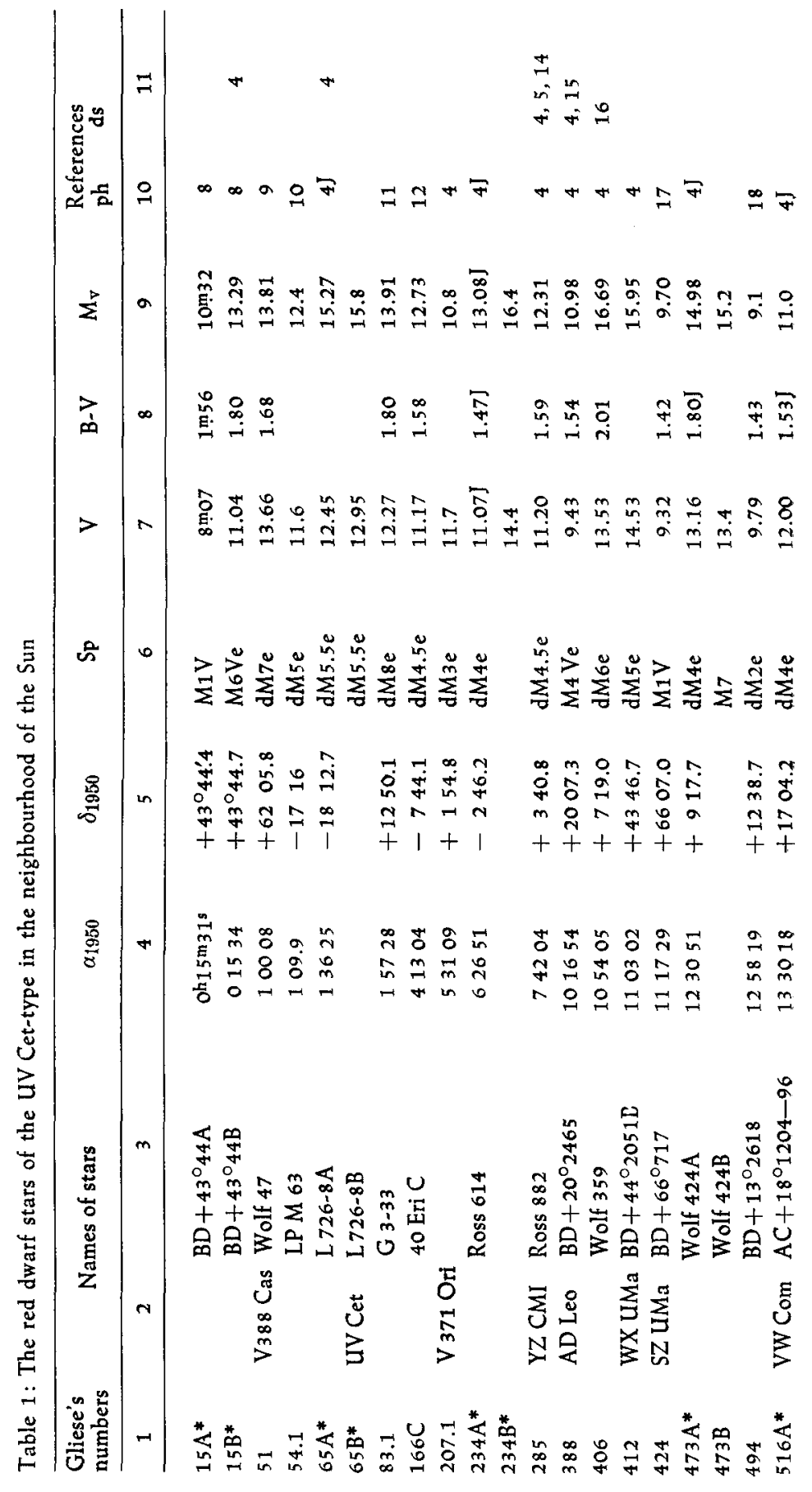




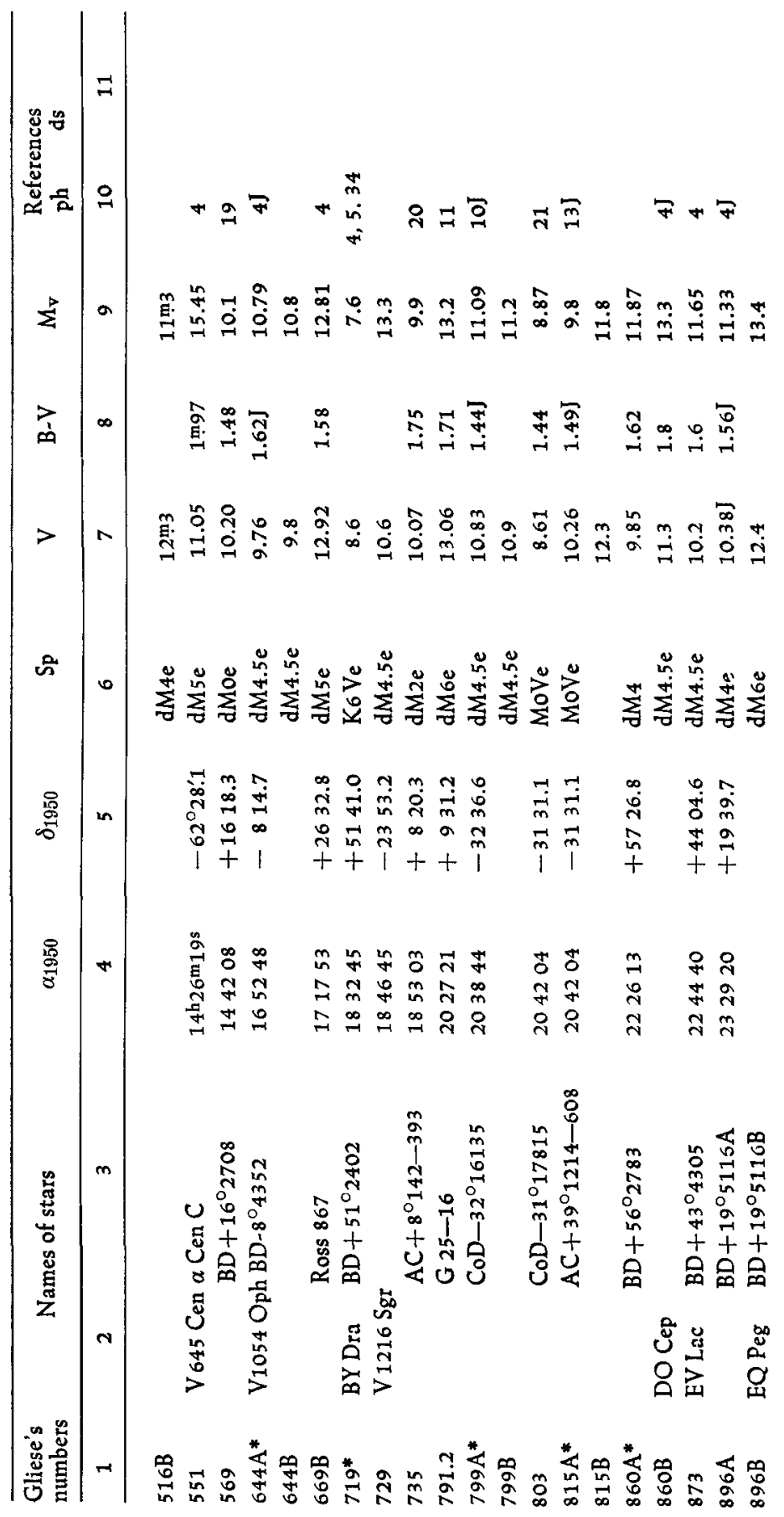


Notes to Table 1.

$15 \mathrm{AB}: \mathrm{P}=3020 y, \mathrm{a}=43$ ".94 (7).

$15 \mathrm{~A}$ is $\mathrm{SB}$, the hydrogen emission was not observed, the Ca II emission is very weak (B).

15B: the hydrogen emission was not observed in the quict state spectra, the Ca II emission is very weak and variable.

$$
\begin{aligned}
& 65 \mathrm{AB}: \mathrm{P}=200 \% .0, \mathrm{a}=51.570 \text { (7). } \\
& \text { 234AB: } \mathrm{P}=16 \text { \%.50, } \mathrm{a}=\mathrm{O}^{\prime \prime} .980 \text { (7). } \\
& 475 \mathrm{AB}: \varrho=1 \text { ". } 0, \Theta=132^{\circ}{ }_{1} \text { (1938); } \varrho=0 \text { ". } 8 \text { (1960), } \\
& \Theta=169^{\circ}(1960)(7) \text {. }
\end{aligned}
$$

$516 \mathrm{AB}: \varrho=3$ ". $\mathrm{o}, \Theta=22^{\circ}(7)$.

$644 \mathrm{AB}: \mathrm{P}=1$ y.72, $\mathrm{a}=\mathrm{O}^{\prime \prime} 218(7)$.

719 is $\mathrm{SB}: \mathrm{P}=5 \mathrm{~d} 981$. Except the flare activity, the periodical light variability was observed with $P=3$ d 836 (5).

$799 \mathrm{AB}: \varrho=2^{\prime \prime}, \Theta=231^{\circ}(7)$.

$815 \mathrm{AB}: \varrho=\mathrm{o}^{\prime \prime} 1, \Theta=81 \% \circ(7)$.

$860 \mathrm{AB}: \mathrm{P}=44 \mathrm{y} 46: \mathrm{a}=2$ ".386 (7). The flares of $\mathrm{B}$ component were observed photographically, during photoelectrical patrol the components were observed together.

$896 \mathrm{AB}: \mathrm{P}=177 \mathrm{y} .90, \mathrm{a}=2$ ". $386(7)$.

Several stars included in previous lists of UV Ceti-type objects do not fit our determination of this kinds of variables because photometric observations of brightness variations of the K-M dwarfs do not permit to construct the flare light curves, and we do not consider observations of emission line variability as features which allow to attribute the variable to UV Ceti-type. These stars were included in Table II as the suspected UV Ceti-type variables. The stars from Table II show the brightness or spectral variability which may be explained by the flare activity. The most of stars in this list have no precise spectral classification. The most of the brightness variability observations was made photographically, and there are no data to construct the flare light curves. The spectral variability consists of appearance or strengthening of the emission lines on some spectrograms.

It is possible that all $\mathrm{K}-\mathrm{M}$ drawfs with emission lines in quiet spectra are UV Ceti-type variables. In present time only 35 stars of $65 \mathrm{M}$-type known dwarfs with hydrogen emission (25) have shown the flare activity and the brightness or spectral variability. The special observations of remaining stars from the list (25) are desirable to dheck their membership to UV Cet-type variables. But red dwarfs with emission in spectra and without brightness or spectral variability are not included in Table II.

It is possible that a part of stars in Table II belongs to UVn-type variables (flash stars) the flare stars of stellar aggregates similar to those in Taurus and Orion. The flare star belonging to such aggregates certainly were not included in Table II.

The Table II includes the following data on the suspected UV Cet-type stars: 1) the number; 2) the designation from $(5,6) ; 3)$ the number from other catalogues; 4-5) Right ascension and declination for 1950.0 ; 6) spectral type; 7) $\mathrm{m}$ - the magnitudes: $\mathrm{V}$ - visual in the UBV-system and $p$ - photographic. All data in the columns 4-7 are taken from (5-7) or from the original investigation. In two last columns the bibliographic information is given. An asterisk in the column 1 means that there is a note at the end of the Table II. 
Table II: The suspected UV-type variable stars

\begin{tabular}{|c|c|c|c|c|c|c|c|c|}
\hline \multirow{3}{*}{1} & \multirow{3}{*}{$\begin{array}{l}\begin{array}{l}\text { GCVS } \\
\text { or Gl. }\end{array} \\
\text { V Psc }\end{array}$} & \multirow{3}{*}{$\begin{array}{c}\text { Other } \\
\text { catalogues }\end{array}$} & \multirow{3}{*}{$\frac{\mathrm{RA}_{(1950.0)}}{\text { oh12m49s }^{\text {m }}}$} & \multirow{3}{*}{$\begin{array}{c}\text { Decl. } \\
+6^{\circ} 23^{\prime} .8\end{array}$} & \multirow{3}{*}{$\frac{\text { Sp. }}{M 1 V}$} & \multirow{3}{*}{$\frac{\mathrm{m}}{12^{\mathrm{m} p}}$} & \multirow{2}{*}{\multicolumn{2}{|c|}{$\begin{array}{l}\text { Bibliography } \\
\text { ph sp }\end{array}$}} \\
\hline & & & & & & & & \\
\hline & & & & & & & 5 & \\
\hline 2 & & SPZ 1729 & 04002 & +4014.2 & & $16.8 \mathrm{p}$ & 23 & \\
\hline 3 & QZ Per & & 31445 & +3723.2 & & $17.5 p$ & 5 & \\
\hline $4^{*}$ & II Tau & HII 2411 & 34643 & +2410.0 & M4e & $15.5 \mathrm{p}$ & 5,24 & \\
\hline $5^{*}$ & IN Tau & & 42106 & +1450 & M3e & $15.3 p$ & 5 & \\
\hline 6* & IO Tau & & 42124 & +1458 & M & $16.7 p$ & 5 & \\
\hline $7^{*}$ & PZ Mon & $\mathrm{BD}+1^{\circ} 1522$ & 64546 & +116.6 & $\mathrm{~K} 2 \mathrm{Ve}$ & $10.8 \mathrm{p}$ & 5,34 & \\
\hline $8^{*}$ & & $\mathrm{BD}+33^{\circ} 1646 \mathrm{~B}$ & 80541 & +3256 & $\mathrm{dMe}$ & $11 \mathrm{p}$ & & 25 \\
\hline 9 & DH Car & & 111245 & -6129.3 & & $14.9 p$ & 4,5 & \\
\hline $10^{*}$ & $\mathrm{Gl} 451 \mathrm{~B}$ & $\mathrm{BD}+38^{\circ} 3385 \mathrm{~B}$ & 115006 & +3804.6 & & & 26 & \\
\hline 11 & & $\mathrm{G}_{177.4}$ & 125342 & +5112.1 & & $16.1 p$ & 27 & \\
\hline $12^{*}$ & & & 125512 & -6533 & & $14.7 p$ & & 28 \\
\hline $13^{*}$ & & & 130230 & -6156 & & $12.7 p$ & & 28 \\
\hline $14^{*}$ & & & 133008 & -6158 & & $15.5 p$ & & 28 \\
\hline $15^{*}$ & Gl 526 & $\mathrm{BD}+15^{\circ} 2620$ & 134312 & +1509.7 & $\mathrm{dM} 4 \mathrm{e}$ & $8.50 \mathrm{~V}$ & & 29 \\
\hline $16^{*}$ & Gl 616.2 & $\mathrm{BD}+55^{\circ} 1823$ & 161559 & +5523.8 & dM1e & $9.96 \mathrm{~V}$ & 4 & \\
\hline 17 & V475Her & & 171804 & +2515 & & $15.8 \mathrm{p}$ & 5 & \\
\hline 18 & & G258-7 & 172725 & +6701.0 & & $16.3 p$ & 30 & \\
\hline 19 & V2354 Sgr & & 182704 & -2453.5 & & $13.2 p$ & 5 & \\
\hline $20^{*}$ & Gl752 & $\mathrm{BD}+4^{\circ} 4048 \mathrm{~B}$ & 191432 & +504.7 & $\mathrm{dM} 5 \mathrm{e}$ & $17.38 \mathrm{~V}$ & 4,31 & \\
\hline 21 & FV Vul & & 193639 & +2729.0 & $M:$ & $14.1 p$ & 5 & \\
\hline 22 & KO Vul & & 195519 & +2857 & & $17.5 p$ & 5 & \\
\hline 23 & Gl 781 & Wolf 1130 & 200355 & +5418.2 & $\mathrm{~d} M 3 \mathrm{e}$ & $11.9 \mathrm{~V}$ & 4 & \\
\hline $24^{*}$ & & & 2004.9 & +6627 & & $17.9 \mathrm{p}$ & 32 & \\
\hline $25 *$ & & & 2013.0 & +6747 & & $17.7 \mathrm{p}$ & 32 & \\
\hline $26^{*}$ & & & 2041.4 & +6659 & & $18.0 \mathrm{p}$ & 32 & \\
\hline $27^{*}$ & & & 2041.7 & +6701 & & $18.0 \mathrm{p}$ & 32 & \\
\hline $28^{*}$ & & & 2044.5 & +6643 & & $18.0 p$ & 32 & \\
\hline $29^{*}$ & & & 2046 & +6659.7 & & $18.0 p$ & 32 & \\
\hline $30^{*}$ & & & 2046 & +6710 & & $20.5 p$ & 32 & \\
\hline $31^{*}$ & & & 2049 & +6739.5 & & $17.1 \mathrm{p}$ & 33 & \\
\hline $32^{*}$ & & & 2054.9 & +6815 & & 18.2 & 32 & \\
\hline 33 & AK Mic & & 210328 & -4014.0 & & $16.2 p$ & 5 & \\
\hline $34^{*}$ & Gl 867B & $\mathrm{BD}-21^{\circ} 6267 \mathrm{~B}$ & 223601 & -2052.8 & $\mathrm{~d} M 4 \mathrm{e}$ & $11.45 \mathrm{~V}$ & & 25 \\
\hline 35 & FZ And & & 230319 & +5251.4 & & 15.5 & 5 & \\
\hline
\end{tabular}

Notes to Table II.

4. 61 Hares were observed photographically. A possible member of Hyades cluster (24).

5. A possible member of Hyades cluster (5).

6. A possible member of Hyades cluster (5).

7. The flare was observed photographically in B-photometric system $\left(\Delta \mathrm{mB}_{\mathrm{B}}=\mathrm{om} 11\right)$ (34). Possible UVn-type (flash) star (4).

8. Dyer suspects the variability of this star (25).

10. The star $\mathrm{BD}+38^{\circ} 2285$ has a flare component, which is normally unseen. The flare was observed photographically (26). 
12.-14. SANDULEAK observed the strengthening of emission lines. The possible UVntype stars.

15. The variability of Call emission was detected in (29). The hydrogen emission was not observed.

16. The light variability was detected photographically, the star was monitored for $17 \mathrm{~h}_{5}$ in B system with no flares detected (34).

20. HERBIG has observed the appearance of the hydrogen and $\mathrm{Ca}$ II emission at one of three spectrograms (31).

24-32. The flares were observed by photographical search for the UVn-type variables in the nebula NGC 7023. All these stars dispose far off the nebula and are the background stars (32).

34. This star is about $\mathbf{8}^{\prime \prime}$ distance from its primary. Very strong hydrogen and Ca Il emission lines are present on spectrograms taken at Mount Wilson by DYER (25).

$$
\text { Ref erences: }
$$

(1) A. JOY, 1960, „Stellar atmopheres“, ed. by J. L. Greenstein, Chicago.

(2) V. S. OSKAN JAN, 1964, Publ. Beograd Astr. Obs. N 10, 1964.

(3) L. H. SOLOMON, Smithsonian Astrophys. Obs. Special Rep. N 210.

(4) R. E. GERSHBERG, 1970, "The flares of the red dwarf stars“. Nauka. Moscow.

(5) B. V. KUKARKIN et al., 1969, General Catalogue of Variable Stars. Moscow.

(6) W. GLIESE, 1969, Veröff, des Astron. Rechen-Instituts Heidelberg N22.

(7) R. WOOLEY, E. A. EPPS, M. J. PENSTON and S. B. POCOCK, 1970, Roy. Obs. Ann. N 5.

(8) N. I. SHAKHOVSKAYA, Izv. Crimean Astrophys. Obs. 47.

(9) H. L. JOHNSON, W. W. MORGAN, 1953, Aph. J. 117, 313.

(10) W. E. KUNKEL, 1970, IBVS N 442.

(1i) W. E. KUNKEL, 1968, IBVS N 294.

(12) W. E. KUNKEL, Low-Luminosity Stars“ed. by S. S. Kumar, p. 195

(13) N. I. SHAKHOVSKAYA, Izy. Crimean Astrophys. Obs. 45.

(14) R. E. GERSHBERG, Izv. Crimean Astrophys. Obs. 45.

(15) R. E. GERSHBERG, N. I. SHAKHOVSKAYA, 1971, A. J. (USSR) 48

(16) J. L. GREENSTEIN, H. ARP, 1969, Aph. Letters 3, 149.

(17) P. F. CHUGAINOV, Izv. Crimean Astrophys. Obs. 46.

(18) N. I. SHAKHOVSKAYA, 1969, IBVS N 361.

(19) O. EGGEN, 1968, Aph. J. Suppl. N 152, p. 69.

(20) N. I. SHAKHOVSKAYA, K. L. MASLENNIKOV, 1970, IBVS N 487.

(21) G. A. HARDING, 1970, MNASSA 29, N 9, 130.

(22) R. F. GERSHBERG, S. A. KAPLAN, Izv. Crimean Astrophys. Obs. 44.

(23) A. S. SHAROV, A. K. ALKSNIS, 1970, Astron. Zirk. USSR N 600.

(24) V. A. AMBARTSUMIAN, L. V. MIRZOYAN, E. S. PARSAMIAN, H. S. CHAVUSHIAN, L. K. ERASTOVA, 1971, Byurakan Astrophys. Obs. Preprint N 2.

(25) W. P. BIDELMAN, 1954, Aph. J. Suppl. N 7, p. 216.

(26) P. VAN DE KAMP, ,Low-Luminosity Stars ed. by S. S. Kumar, p. 199.

(27) H. I. GICLAS, R. BURNHAM jr., N. G. THOMAS, 1965, Bull. Lowell Obs. N 129, 203.

(28) N. SANDULEAK, 1969, Aph. J. 155, 1121.

(29) P. KANDEL, 1962, CR 255, 1275.

(30) H. L. GICLAS, R. BURNHAM jr., N. G. THOMAS, 1970, Bull. Lowell Obs. N $152,171$.

(31) G. H. HERBIG, 1956, PASP 68, 531.

(32) E. S PARSAMIAN, 1971, Soobsch. Byurak. Obs., 43, 3.

(33) L. V. MIRZOYAN, E. S. PARSAMIAN, H. S. CHAVUSHIAN, 1968, Soobsch, Byurak. Obs. $39,5$.

'34) S. CRISTALDI, M. RODONO, 1970, Astr. Astrophys. Suppl. 2, 223. 